\title{
POISSON KERNEL FOR THE ASSOCIATED CONTINUOUS $q$-ULTRASPHERICAL POLYNOMIALS
}

\author{
Mizan Rahman and Qazi M. Tariq
}

\begin{abstract}
The bilinear generating function (the Poisson kernel) for the associated continuous $q$-ultraspherical polynomials, first studied by Bustoz and Ismail and later generalized by Ismail and Rahman, is obtained, essentially as a product of a ${ }_{2} \phi_{1}, a_{3} \phi_{2}$, and a ${ }_{4} \phi_{3}$ basic hypergeometric series. Some related generating functions also are given.
\end{abstract}

\section{Introduction}

The continuous $q$-ultraspherical polynomials, $C_{n}(x ; \beta \mid q)$, introduced by Rogers [18, $19,20]$, can be defined by the generating function

$$
\sum_{n=0}^{\infty} C_{n}(x ; \beta \mid q) t^{n}=\frac{\left(\beta t e^{i \theta}, \beta t e^{-i \theta} ; q\right)_{\infty}}{\left(t e^{i \theta}, t e^{-i \theta} ; q\right)_{\infty}}
$$

where $0<q<1, x=\cos \theta(0 \leq \theta \leq \pi),|t|<1$, and

$$
\begin{aligned}
(a ; q)_{\infty} & =\prod_{j=0}^{\infty}\left(1-a q^{j}\right), \\
\left(a_{1}, a_{2}, \ldots, a_{k} ; q\right)_{\infty} & =\prod_{j=1}^{k}\left(a_{j} ; q\right)_{\infty}
\end{aligned}
$$

see [7]. Setting $\beta=0$ in (1.1), one obtains the generating function for the continuous $q$-Hermite polynomials $H_{n}(x \mid q)=(q ; q)_{n} C_{n}(x ; 0 \mid q)$ :

$$
\sum_{n=0}^{\infty} \frac{H_{n}(x \mid q)}{(q ; q)_{n}} t^{n}=\frac{1}{\left(t e^{i \theta}, t e^{-i \theta} ; q\right)_{\infty}}
$$

where the shifted factorial $(a ; q)_{n}$ is defined by

$$
\begin{aligned}
& (a ; q)_{0}=1, \\
& (a ; q)_{n}=\frac{(a ; q)_{\infty}}{\left(a q^{n} ; q\right)_{\infty}}=\prod_{j=1}^{n}\left(1-a q^{j-1}\right) \quad n=1,2, \ldots
\end{aligned}
$$

Received November 13, 1996, revised January 20, 1997.

1991 Mathematics Subject Classification: 33A65.

Key words and phrases: associated Askey-Wilson polynomials, associated continuous $q$-ultraspherical polynomials, generating functions, bilinear generating function, nonsymmetric bilinear generating function. 
As a $q$-extension of Mehler's famous bilinear generating function for the classical Hermite polynomials,

$$
\sum_{n=0}^{\infty} \frac{H_{n}(x) H_{n}(y)}{n !}\left(\frac{t}{2}\right)^{n}=\left(1-t^{2}\right)^{-\frac{1}{2}} \exp \left\{\frac{2 x y t-\left(x^{2}+y^{2}\right) t^{2}}{1-t^{2}}\right\},
$$

see [21, Problem 23] and [22], Rogers [17] found the following extension of (1.5) for the $q$-Hermite polynomials:

$$
\sum_{n=0}^{\infty} \frac{H_{n}(x \mid q) H_{n}(y \mid q)}{(q ; q)_{n}} t^{n}=\frac{\left(t^{2} ; q\right)_{\infty}}{\left|\left(t e^{i(\theta+\varphi)}, t e^{i(-\theta+\varphi)} ; q\right)_{\infty}\right|^{2}}
$$

$x=\cos \theta, y=\cos \varphi,|t|<1$. The extension of (1.5) to $q$-ultraspherical polynomials was found by Gasper and Rahman [8]:

$$
\begin{aligned}
P_{t}(x, y ; \beta \mid q):= & \sum_{n=0}^{\infty} h_{n} C_{n}(x ; \beta \mid q) C_{n}(y ; \beta \mid q) t^{n} \\
= & \frac{(q, \beta q ; q)_{\infty}}{2 \pi\left(\beta, \beta t^{2} ; q\right)_{\infty}}\left|\frac{\left(\beta t e^{i \theta+i \varphi}, \beta t e^{i \theta-i \varphi} ; q\right)_{\infty}}{\left(t e^{i \theta+i \varphi}, t e^{i \theta-i \varphi} ; q\right)_{\infty}}\right|^{2} \\
& \times{ }_{8} W_{7}\left(\beta t^{2} / q ; \beta / q, t e^{i \theta+i \varphi}, t e^{-i \theta-i \varphi}, t e^{i \theta-i \varphi}, t e^{-i \theta+i \varphi} ; q, \beta q\right),
\end{aligned}
$$

$\beta, t$ assumed real, where

$$
h_{n}=\frac{\left(q, \beta^{2} ; q\right)_{\infty}}{2 \pi(\beta, \beta q ; q)_{\infty}} \frac{\left(1-\beta q^{n}\right)(q ; q)_{n}}{(1-\beta)\left(\beta^{2} ; q\right)_{n}}
$$

is the normalizing constant in the orthogonality relation

$$
\int_{0}^{\pi} C_{n}(\cos \theta ; \beta \mid q) C_{m}(\cos \theta ; \beta \mid q) W_{\beta}(\cos \theta \mid q) d \theta=\frac{\delta_{m, n}}{h_{n}(\beta \mid q)}
$$

with

$$
W_{\beta}(\cos \theta \mid q)=\frac{\left(e^{2 i \theta}, e^{-2 i \theta} ; q\right)_{\infty}}{\left(\beta e^{2 i \theta}, \beta e^{-2 i \theta} ; q\right)_{\infty}}
$$

see [7] and [3], and ${ }_{8} W_{7}$ is a special case of a very-well-poised basic hypergeometric series:

$$
\begin{aligned}
& { }_{2 m} W_{2 m-1}\left(a ; a_{1}, a_{2}, \ldots, a_{2 m-3} ; q, z\right) \\
& \quad:={ }_{2 m} \phi_{2 m-1}\left[\begin{array}{c}
a, q \sqrt{a},-q \sqrt{a}, a_{1}, a_{2}, \ldots, a_{2 m-3} \\
\sqrt{a},-\sqrt{a}, q a / a_{1}, q a / a_{2}, \ldots, q a / a_{2 m-3}
\end{array} ; q, z\right]
\end{aligned}
$$

where the symbol on the right-hand side is a special case of a basic hypergeometric series defined by

$$
{ }_{r+1} \phi_{r}\left[\begin{array}{c}
a_{1}, a_{2}, \ldots, a_{r+1} \\
b_{1}, b_{2}, \ldots, b_{r}
\end{array} ; q, z\right]:=\sum_{k=0}^{\infty} \frac{\left(a_{1} \ldots a_{r+1} ; q\right)_{k}}{\left(q, b_{1}, \ldots, b_{r} ; q\right)_{k}} z^{k}
$$


where

$$
\left(a_{1}, \ldots, a_{j} ; q\right)_{k}=\prod_{i=1}^{j}\left(a_{i} ; q\right)_{k}
$$

see [7] for conditions of convergence and other properties of these series. One can see that the Poisson kernel in (1.7) reduces to (1.6) when $\beta=0$, except for a constant factor. Formula (1.7) is admittedly more complicated than (1.6), but it does exhibit one of the basic properties of the Poisson kernel, namely, the positivity in some domain of $\beta$ and $t$. The importance of positivity of these bilinear sums in Fourier analysis was pointed out by many authors, see, for example, Askey [1]. But there is renewed interest in Poisson kernels of orthogonal polynomials for a different reason. Wiener [23] showed in 1933 how the classical topic of Fourier integrals could be approached from the point of view of classical orthogonal polynomials, in particular, by using Mehler's formula (1.5). One can show that the expression on the right-hand side of (1.5) approaches a multiple of $\exp (i x y)$, the usual kernel of Fourier transform, as $t \rightarrow i$ on the unit circle. This was suggested by Askey as a suitable starting point for a systematic $q$-extension of Fourier Analysis, which resulted in a number of recent publications $[2,4,15]$. It turns out that the most important piece of information that one needs from the Poisson kernel is the location of poles in the complex $t$-plane, as far as the Fourier transformation is concerned. One can see that the poles of the kernel in (1.7) are exactly the same as those of the simpler kernel in (1.6). Calculations with more general systems of polynomials, starting with the 2-parameter continuous $q$-Jacobi polynomials [9] up to the 4-parameter Askey-Wilson polynomials [16] reveal that this phenomenon persists through the entire Askey-Wilson family. Let us recall that the Askey-Wilson polynomials are defined by [3]

$$
p_{n}(x ; a, b, c, d \mid q)={ }_{4} \phi_{3}\left[\begin{array}{c}
q^{-n}, a b c d q^{n-1}, a e^{i \theta}, a e^{-i \theta} \\
a b, a c, a d
\end{array} ; q, q\right],
$$

$x=\cos \theta$, which satisfy an orthogonality relation similar to (1.9) if $\max (|a|,|b|,|c|,|d|)$ $<1$. Note that $C_{n}(x ; \beta \mid q)$ is a special case of (1.13) (except for a constant factor) that corresponds to setting $a=\sqrt{\beta}, b=\sqrt{\beta q}, c=-\sqrt{\beta}, d=-\sqrt{\beta q}$; see $[3,5,7]$. In this paper, we wish to generalize (1.7) in a different direction. Recall that $C_{n}(x ; \beta \mid q)$ satisfies the three-term recurrence relation

$$
2 x C_{n}(x ; \beta \mid q)=\frac{1-q^{n+1}}{1-\beta q^{n}} C_{n+1}(x ; \beta \mid q)+\frac{1-\beta^{2} q^{n-1}}{1-\beta q^{n}} C_{n-1}(x ; \beta \mid q)
$$

with $C_{-1}(x ; \beta \mid q)=0, C_{0}(x ; \beta \mid q)=1,-1 \leq x \leq 1$. Bustoz and Ismail [6] introduced an association parameter $\alpha$ and studied the properties of the associated $q$-ultraspherical polynomial $C_{n}^{\alpha}(x ; \beta \mid q)$ that satisfies the recurrence relation

$$
2 x C_{n}^{\alpha}(x ; \beta \mid q)=\frac{1-q^{n+\alpha+1}}{1-\beta q^{n+\alpha}} C_{n+1}^{\alpha}(x ; \beta \mid q)+\frac{1-\beta^{2} q^{n+\alpha-1}}{1-\beta q^{n+\alpha}} C_{n-1}^{\alpha}(x ; \beta \mid q)
$$

with $C_{-1}^{\alpha}(x ; \beta \mid q)=0, C_{0}^{\alpha}(x ; \beta \mid q)=1$. They found a measure $d \mu(x)$ with respect to which the orthogonality relation

$$
\int_{-1}^{1} C_{n}^{\alpha}(x ; \beta \mid q) C_{m}^{\alpha}(x ; \beta \mid q) d \mu(x)=\frac{1-\beta q^{\alpha}}{1-\beta q^{\alpha+n}} \frac{\left(\beta^{2} q^{\alpha} ; q\right)_{n}}{\left(q^{\alpha+1} ; q\right)_{n}} q^{\alpha n} \delta_{m, n}
$$


holds. By a straightforward manipulation of the recurrence relation (1.15), Bustoz and Ismail [6] also found the generating function

$$
\begin{aligned}
& G_{t}^{\alpha}(x ; \beta \mid q):=\sum_{n=0}^{\infty} C_{n}^{\alpha}(x ; \beta \mid q) t^{n} \\
& =\frac{\left(1-q^{\alpha}\right)}{\left(1-2 x t+t^{2}\right)}{ }_{3} \phi_{2}\left[\begin{array}{c}
\beta t e^{i \theta}, \beta t e^{-i \theta}, q \\
q t e^{i \theta}, q t e^{-i \theta},
\end{array} ; q, q^{\alpha}\right] .
\end{aligned}
$$

In Section 4, we will find an alternative form of (1.17), as well as a few other generating functions. In Section 5, we will compute some bilinear generating functions. The main result of this paper that follows from (5.1) and (5.5) is

$$
\begin{aligned}
K_{t}^{\alpha}(x, y ; \beta \mid q): & =\sum_{n=0}^{\infty} \frac{\left(1-\beta q^{\alpha+n}\right)}{\left(1-\beta q^{\alpha}\right)} \frac{\left(q^{\alpha+1} ; q\right)_{n}}{\left(\beta^{2} q^{\alpha} ; q\right)_{n}}\left(t q^{-\alpha}\right)^{n} C_{n}^{\alpha}(x ; \beta \mid q) C_{n}^{\alpha}(y ; \beta \mid q) \\
& =\left(1-\beta q^{\alpha}\right)^{-1}\left\{L_{t}^{\alpha}(x, y)-\beta q^{\alpha} L_{t q}^{\alpha}(x, y)\right\}
\end{aligned}
$$

where

$$
\begin{aligned}
L_{t}^{\alpha}(x, y)= & \frac{\left(1-q^{\alpha}\right)^{2}}{\left(1-e^{-2 i \theta}\right)\left(1-2 y t q^{-\alpha} e^{i \theta}+t^{2} q^{-2 \alpha} e^{2 i \theta}\right)} \frac{\left(q^{\alpha+1} ; q\right)_{\infty}}{\left(\beta^{2} q^{\alpha} ; q\right)_{\infty}} \\
& \times{ }_{2} \phi_{1}\left[\begin{array}{c}
\beta e^{-2 i \theta}, \beta \\
q e^{-2 i \theta} ; q, q^{\alpha}
\end{array}\right] \\
& \times{ }_{3} \phi_{2}\left[\begin{array}{c}
\beta t q^{-\alpha} e^{i(\theta+\varphi)}, \beta t q^{-\alpha} e^{i(\theta-\varphi)}, q \\
\left.t q^{1-\alpha} e^{i(\theta+\varphi)}, t q^{1-\alpha} e^{i(\theta-\varphi)} ; q, q^{\alpha}\right]
\end{array}\right. \\
& \times{ }_{4} \phi_{3}\left[\begin{array}{l}
\beta e^{2 i \theta}, \beta, t q^{-\alpha} e^{i(\theta+\varphi)}, t q^{-\alpha} e^{i(\theta-\varphi)} \\
\left.q e^{2 i \theta}, \beta t q^{-\alpha} e^{i(\theta+\varphi)}, \beta t q^{-\alpha} e^{i(\theta-\varphi)} ; q, q\right]
\end{array}\right. \\
+ & (\theta \longleftrightarrow-\theta) .
\end{aligned}
$$

Note that $K_{t}^{\alpha}$ is a multiple of the Poisson kernel for $C_{n}^{\alpha}(x ; \beta \mid q)$. This shows that the poles are only slightly shifted by a factor of $q^{\alpha}$, but otherwise remains the same as in (1.7). Unfortunately, we were unable to simplify (1.19) to a form where the positivity would become obvious as it is in (1.7). It is only in the case when $\alpha=0$ that the two ${ }_{4} \phi_{3}$ series in (1.19) combine to an ${ }_{8} \phi_{7}$ which is what we have in (1.7), but otherwise the computation seems quite difficult.

In deriving the formulas mentioned above, our main tool has been the generating function (1.17) as well as the following moment representation of $C_{n}^{\alpha}(x ; \beta \mid q)$ :

$$
C_{n}^{\alpha}(x ; \beta \mid q)=\int_{-\infty}^{\infty} \xi^{n} d \nu(\xi ; \beta, x)
$$


where $\nu$ is a step function whose jumps occur at the points $q^{k} e^{i \theta}$ and $q^{k} e^{-i \theta}, k=$ $0,1, \ldots$ The jumps are

$$
\begin{aligned}
d \nu\left(q^{k} e^{i \theta} ; \beta, x\right)= & \frac{\left(1-q^{\alpha}\right)}{\left(1-e^{-2 i \theta}\right)}{ }^{2} \phi_{1}\left[\begin{array}{c}
\beta e^{-2 i \theta}, \beta \\
q e^{-2 i \theta} ; q, q^{\alpha}
\end{array}\right] \\
& \times \frac{\left(q e^{2 i \theta} / \beta, q / \beta ; q\right)_{k}}{\left(q, q e^{2 i \theta} ; q\right)_{k}}\left(\beta^{2} q^{\alpha}\right)^{k},
\end{aligned}
$$

with $d \nu\left(q^{k} e^{-i \theta} ; \beta, x\right)$ the same as $(1.21)$ and with $\theta$ replaced by $-\theta$. We prove this important result in Section 3. The idea of this moment representation of orthogonal polynomials is not new. Karlin [13] used such a representation to study the sign regularity of determinants of classical orthogonal polynomials. Carlitz and Al-Salam used them in the 1960s (see the references in [11] and [12]). Ismail and Stanton [11] constructed several moment representations explicitly with special emphasis on the Al-Salam-Chihara and continuous $q$-ultraspherical polynomials. Implicit use of moment representations of the continuous $q$-ultraspherical polynomials was also there in formulas (7.4.4) and (7.4.7) of [7]. The derivation of (1.20) and (1.21) was facilitated by an alternate form of the polynomial solution of the three-term recurrence relation for the associated Askey-Wilson polynomials found in [10]. We carry out this alternate derivation in Section 2. In Section 6, we will give the $q \rightarrow 1$ limit of formulas (1.18) and (1.19), as well as the explicit form of the associated ultraspherical polynomials.

\section{The associated Askey-Wilson polynomials}

In [10], Ismail and Rahman found a representation of the associated Askey-Wilson polynomials, $p_{n}^{\alpha}(x ; a, b, c, d)$, as a linear combination of the two linearly independent solutions of the three-term recurrence relation

$$
\begin{aligned}
& 2 x p_{n}^{\alpha}(x ; a, b, c, d)=A_{n+\alpha} p_{n+1}^{\alpha}(x ; a, b, c, d) \\
& \quad+B_{n+\alpha} p_{n}^{\alpha}(x ; a, b, c, d)+C_{n+\alpha} p_{n-1}^{\alpha}(x ; a, b, c, d), \\
& p_{-1}^{\alpha}(x ; a, b, c, d)=0 \text { and } p_{0}^{\alpha}(x ; a, b, c, d)=1, \text { namely }, \\
& \left.r_{\alpha+n}(z ; a, b, c, d)=\frac{\left(a b q^{\alpha+n}, a c q^{\alpha+n}, a d q^{\alpha+n}, b c d q^{\alpha+n} / z ; q\right)_{\infty}}{\left(b c q^{\alpha+n}, b d q^{\alpha+n}, c d q^{\alpha+n}, a z q^{\alpha+n} ; q\right)_{\infty}} \frac{a}{z}\right)^{n+\alpha} \\
& \quad \times{ }_{8} W_{7}\left(b c d / z q ; b / z, c / z, d / z, a b c d q^{\alpha+n-1}, q^{-\alpha-n} ; q, q z / a\right)
\end{aligned}
$$

and

$$
\begin{aligned}
& s_{\alpha+n}(z ; a, b, c, d) \\
& =\frac{\left(a b c d q^{2 \alpha+2 n}, b z q^{\alpha+n+1}, c z q^{\alpha+n+1}, d z q^{\alpha+n+1}, b c d z q^{\alpha+n} ; q\right)_{\infty}}{\left(b c q^{\alpha+n}, b d q^{\alpha+n}, c d q^{\alpha+n}, q^{\alpha+n+1}, b c d z q^{2 \alpha+2 n+1} ; q\right)_{\infty}}(a z)^{n+\alpha} \\
& \quad \times{ }_{8} W_{7}\left(b c d z q^{2 \alpha+2 n} ; b c q^{\alpha+n}, b d q^{\alpha+n}, c d q^{\alpha+n}, q^{\alpha+n+1}, q z / a ; q, a z\right)
\end{aligned}
$$


where $z=e^{i \theta}, x=\cos \theta$, and

$$
\begin{aligned}
& A_{\alpha}=a^{-1} \frac{\left(1-a b q^{\alpha}\right)\left(1-a c q^{\alpha}\right)\left(1-a d q^{\alpha}\right)\left(1-a b c d q^{\alpha-1}\right)}{\left(1-a b c d q^{2 \alpha-1}\right)\left(1-a b c d q^{2 \alpha}\right)} \\
& C_{\alpha}=a \frac{\left(1-b c q^{\alpha-1}\right)\left(1-b d q^{\alpha-1}\right)\left(1-c d q^{\alpha-1}\right)\left(1-q^{\alpha}\right)}{\left(1-a b c d q^{2 \alpha-1}\right)\left(1-a b c d q^{2 \alpha-2}\right)} \\
& B_{\alpha}=a+a^{-1}-A_{\alpha}-C_{\alpha} .
\end{aligned}
$$

One can show by using Bailey's transformation formula [7, III.(37)] for very-wellpoised ${ }_{8} \phi_{7}$ series that $r_{\alpha+n}(z ; a, b, c, d)$ is a linear combination of $s_{\alpha+n}(z ; a, b, c, d)$ and $s_{\alpha+n}\left(z^{-1} ; a, b, c, d\right)$ with coefficients independent of $n$. But, of course, $s_{\alpha+n}(z ; a, b, c, d)$ and $s_{\alpha+n}\left(z^{-1} ; a, b, c, d\right)$ are also independent solutions of (2.1) as long as $0<\theta<\pi$, so one might seek a representation of the form

$$
p_{n}^{\alpha}(x ; a, b, c, d)=L_{\alpha} s_{\alpha+n}(z ; a, b, c, d)+M_{\alpha} s_{\alpha+n}\left(z^{-1} ; a, b, c, d\right) .
$$

The initial conditions then fix the coefficient $L_{\alpha}$ and $M_{\alpha}$ :

$$
\begin{aligned}
& p_{n}^{\alpha}(x ; a, b, c, d) \\
& =\frac{s_{\alpha+n}(z ; a, b, c, d) s_{\alpha-1}\left(z^{-1} ; a, b, c, d\right)-s_{\alpha+n}\left(z^{-1} ; a, b, c, d\right) s_{\alpha-1}(z ; a, b, c, d)}{s_{\alpha}(z ; a, b, c, d) s_{\alpha-1}\left(z^{-1} ; a, b, c, d\right)-s_{\alpha}\left(z^{-1} ; a, b, c, d\right) s_{\alpha-1}(z ; a, b, c, d)} .
\end{aligned}
$$

Using (2.1) and an argument similar to the one used in [10], we can show that

$$
\begin{aligned}
& \left\{s_{\alpha}(z ; a, b, c, d) s_{\alpha-1}\left(z^{-1} ; a, b, c, d\right)-s_{\alpha}\left(z^{-1} ; a, b, c, d\right) s_{\alpha-1}(z ; a, b, c, d)\right\}^{-1} \\
& =\frac{\left(b c q^{\alpha-1}, b d q^{\alpha-1}, c d q^{\alpha-1}, q^{\alpha} ; q\right)_{\infty}}{\left(a b q^{\alpha}, a c q^{\alpha}, a d q^{\alpha}, a b c d q^{\alpha-1} ; q\right)_{\infty}} \\
& \quad \times \frac{a^{1-2 \alpha}}{\left(1-a b c d q^{2 \alpha-2}\right)} \frac{(a z, a / z ; q)_{\infty}}{\left(q z^{2}, q / z^{2} ; q\right)_{\infty}} \frac{1}{\left(z-z^{-1}\right)}
\end{aligned}
$$

which is just the Casorati determinant of (2.1). Using the transformation formula [7, III (23)] and substituting (2.9) in (2.8), we obtain

$$
\begin{aligned}
& p_{n}^{\alpha}(x ; a, b, c, d)=\frac{\left(1-a b c d q^{\alpha-2}\right)}{\left(1-a b c d q^{2 \alpha-2}\right)} \\
& \times \frac{\left(a q^{\alpha} / z, b q^{\alpha} / z, c q^{\alpha} / z, d q^{\alpha} / z, a z q^{\alpha+n+1}, b z q^{\alpha+n+1} ; q\right)_{\infty}}{\left(a b q^{\alpha}, a c q^{\alpha}, a d q^{\alpha}, b c q^{\alpha+n}, b d q^{\alpha+n}, c d q^{\alpha+n} ; q\right)_{\infty}} \\
& \times \frac{\left(c z q^{\alpha+n+1}, d z q^{\alpha+n+1}, a b c d q^{\alpha+n-1} ; q\right)_{\infty}}{\left(q^{\alpha+n+1}, q^{\alpha+1} / z^{2}, z^{2} q^{\alpha+n+2} ; q\right)_{\infty}} \frac{(a z)^{n}}{\left(1-z^{-2}\right)} \\
& \times{ }_{8} W_{7}\left(q^{\alpha} / z^{2} ; q / a z, q / b z, q / c z, q / d z, q^{\alpha} ; q, a b c d q^{\alpha-2}\right) \\
& \times{ }_{8} W_{7}\left(z^{2} q^{\alpha+n+1} ; z q / a, z q / b, z q / c, z q / d, q^{\alpha+n+1} ; q, a b c d q^{\alpha+n-1}\right) \\
&+ z \longleftrightarrow z^{-1} .
\end{aligned}
$$

This is the form that will enable us to represent $C_{n}^{\alpha}(x ; \beta \mid q)$ as the moment of a probability measure. 


\section{Associated q-ultraspherical polynomials as moments}

Using the quadratic transformation formula [7, (3.4.7)], we have

$$
\begin{aligned}
& { }_{8} W_{7}\left(t^{2} q^{\lambda} ; t q / a, t q^{\frac{1}{2}} / a,-t q / a,-t q^{\frac{1}{2}} / a, q^{\lambda} ; q, a^{4} q^{\lambda-1}\right) \\
& \quad=\frac{\left(t^{2} q^{\lambda+1}, a^{4} q^{2 \lambda-1} ; q\right)_{\infty}}{\left(a^{2} q^{\lambda}, a^{2} t^{2} q^{2 \lambda} ; q\right)_{\infty}}{ }_{2} \phi_{1}\left[\begin{array}{c}
q t^{2} / a^{2}, q / a^{2} \\
q t^{2}
\end{array} ; q, a^{4} q^{\lambda-1}\right]
\end{aligned}
$$

see also [14].

Setting $b=a q^{\frac{1}{2}}, c=-a, d=-a q^{\frac{1}{2}}$ in (2.10), using (3.1), and simplifying the coefficient, we obtain

$$
\begin{aligned}
& p_{n}^{\alpha}\left(x ; a, a q^{\frac{1}{2}},-a,-a q^{\frac{1}{2}}\right)=\frac{1-a^{4} q^{\alpha-1}}{1-z^{-2}} \frac{\left(a^{4} q^{\alpha+n} ; q\right)_{\infty}}{\left(q^{\alpha+n+1} ; q\right)_{\infty}}(a z)^{n} \\
& \times{ }_{2} \phi_{1}\left[\begin{array}{c}
q / a^{2} z^{2}, q / a^{2} \\
q / z^{2}
\end{array} ; q, a^{4} q^{\alpha-1}\right] \\
& \times{ }_{2} \phi_{1}\left[\begin{array}{c}
q z^{2} / a^{2}, q / a^{2} \\
q z^{2}
\end{array} ; q, a^{4} q^{\alpha+n}\right] \\
& +z \longleftrightarrow z^{-1} \text {. }
\end{aligned}
$$

Comparing (1.15) with (2.1) for these special values of parameters $b, c, d$, we find that

$$
C_{n}^{\alpha}\left(x ; a^{2} \mid q\right)=\frac{\left(a^{4} q^{\alpha} ; q\right)_{n}}{\left(q^{\alpha+1} ; q\right)_{n}} a^{-n} p_{n}^{\alpha}\left(x ; a, a q^{\frac{1}{2}},-a,-a q^{\frac{1}{2}}\right) .
$$

So, from (3.2) and (3.3), we have

$$
\begin{aligned}
C_{n}^{\alpha}\left(x ; a^{2} \mid q\right)= & \frac{\left(a^{4} q^{\alpha-1} ; q\right)_{\infty}}{\left(q^{\alpha+1} ; q\right)_{\infty}} \frac{z^{n}}{\left(1-z^{-2}\right)}{ }_{2} \phi_{1}\left[\begin{array}{c}
q / a^{2} z^{2}, q / a^{2} \\
q / z^{2}
\end{array} ; q, a^{4} q^{\alpha-1}\right] \\
& \times{ }_{2} \phi_{1}\left[\begin{array}{c}
q z^{2} / a^{2}, q / a^{2} \\
q z^{2}
\end{array} ;, a^{4} q^{\alpha+n}\right] \\
& +z \longleftrightarrow z^{-1} .
\end{aligned}
$$

If we use [7, III.(3)] on the first ${ }_{2} \phi_{1}$ series of the right-hand side, we get

$$
\begin{aligned}
& C_{n}^{\alpha}\left(x ; a^{2} \mid q\right)=\frac{\left(1-q^{\alpha}\right)}{\left(1-z^{-2}\right)} z^{n}{ }_{2} \phi_{1}\left[\begin{array}{c}
a^{2} / z^{2}, a^{2} \\
q / z^{2}
\end{array} ; q, q^{\alpha}\right] \\
& \times{ }_{2} \phi_{1}\left[\begin{array}{c}
q z^{2} / a^{2}, q / a^{2} \\
q z^{2}
\end{array} ; q, a^{4} q^{\alpha+n}\right] \\
& +z \longleftrightarrow z^{-1} \text {. }
\end{aligned}
$$

Since

$$
\lim _{\alpha \rightarrow 0^{+}}\left(1-q^{\alpha}\right)_{2} \phi_{1}\left[\begin{array}{c}
a^{2} / z^{2}, a^{2} \\
q / z^{2}
\end{array} ; q, q^{\alpha}\right]=\frac{\left(a^{2} / z^{2}, a^{2} ; q\right)_{\infty}}{\left(q, q / z^{2} ; q\right)_{\infty}},
$$

one can see that (3.5) is a generalization of $[7,(7.4 .4)]$ as well as of the formula (5.11) of [11]. Replacing $a$ by $\beta^{\frac{1}{2}}$, we find that (1.20) follows from (3.5). 


\section{Some generating functions for $C_{n}^{\alpha}(x ; \beta \mid q)$}

Formula (3.5) is obviously the most convenient form for deriving generating functions for $C_{n}^{\alpha}(x ; \beta \mid q)$. For example, it is easy to see that for any $\lambda$

$$
\begin{aligned}
\sum_{n=0}^{\infty} \frac{(\lambda ; q)_{n}}{(q ; q)_{n}} C_{n}^{\alpha}(x ; \beta \mid q) t^{n}= & \frac{\left(1-q^{\alpha}\right)}{\left(1-z^{-2}\right)} \frac{(\lambda z t ; q)_{\infty}}{(z t ; q)_{\infty}}{ }_{2} \phi_{1}\left[\begin{array}{c}
\beta / z^{2}, \beta \\
q / z^{2}
\end{array} ; q, q^{\alpha}\right] \\
& \times{ }_{3} \phi_{2}\left[\begin{array}{c}
q z^{2} / \beta, q / \beta, z t \\
q z^{2}, \lambda z t
\end{array} ; q, \beta^{2} q^{\alpha}\right] \\
& +z \longleftrightarrow z^{-1} .
\end{aligned}
$$

Assume that $|t|<1$. Setting $\lambda=q$, we get an alternative form of (1.17):

$$
\begin{aligned}
& G_{t}^{\alpha}(x ; \beta \mid q)=\frac{\left(1-q^{\alpha}\right)}{\left(1-z^{-2}\right)(1-z t)}{ }_{2} \phi_{1}\left[\begin{array}{c}
\beta / z^{2}, \beta \\
q / z^{2}
\end{array} ; q, q^{\alpha}\right] \\
& \times{ }_{3} \phi_{2}\left[\begin{array}{c}
q z^{2} / \beta, q / \beta, z t \\
q z^{2}, q z t
\end{array} ; q, \beta^{2} q^{\alpha}\right] \\
& +z \longleftrightarrow z^{-1} \text {. }
\end{aligned}
$$

On the other hand, transforming the second ${ }_{2} \phi_{1}$ series on the right-hand side of (3.5) by $[7$, III.(3)], we get

$$
\begin{aligned}
& C_{n}^{\alpha}\left(x ; a^{2} \mid q\right)=\frac{1-q^{\alpha}}{1-z^{-2}} \frac{\left(q^{\alpha+n+1} ; q\right)_{\infty}}{\left(\beta^{2} q^{\alpha+n} ; q\right)_{\infty}} z_{2}^{n} \phi_{1}\left[\begin{array}{c}
\beta / z^{2}, \beta \\
q / z^{2}
\end{array} ; q, q^{\alpha}\right] \\
& \times{ }_{2} \phi_{1}\left[\begin{array}{c}
\beta z^{2}, \beta \\
q z^{2}
\end{array} ; q, q^{\alpha+n+1}\right] \\
& +z \longleftrightarrow z^{-1} \text {. }
\end{aligned}
$$

Hence,

$$
\begin{aligned}
\sum_{n=0}^{\infty} \frac{\left(q^{\alpha+1} ; q\right)_{n}}{\left(\beta^{2} q^{\alpha} ; q\right)_{n}} C_{n}^{\alpha}(x ; \beta \mid q) t^{n}= & \frac{\left(1-q^{\alpha}\right)}{\left(1-z^{-2}\right)(1-z t)} \frac{\left(q^{\alpha+1} ; q\right)_{\infty}}{\left(\beta^{2} q^{\alpha} ; q\right)_{\infty}} \\
& \times{ }_{2} \phi_{1}\left[\begin{array}{c}
\beta / z^{2}, \beta \\
q / z^{2} ; q, q^{\alpha}
\end{array}\right] \\
& \times{ }_{3} \phi_{2}\left[\begin{array}{c}
\beta z^{2}, \beta, z t \\
q z^{2}, q z t
\end{array} ; q, q^{\alpha+1}\right] \\
+ & z \longleftrightarrow z^{-1} .
\end{aligned}
$$


More generally,

$$
\begin{aligned}
\sum_{n=0}^{\infty} \frac{\left(\lambda, q^{\alpha+1} ; q\right)_{n}}{\left(q, \beta^{2} q^{\alpha} ; q\right)_{n}} C_{n}^{\alpha}(x ; \beta \mid q) t^{n}= & \frac{\left(1-q^{\alpha}\right)}{\left(1-z^{-2}\right)} \frac{\left(q^{\alpha+1} ; q\right)_{\infty}}{\left(\beta^{2} q^{\alpha} ; q\right)_{\infty}} \frac{(\lambda z t ; q)_{\infty}}{(z t ; q)_{\infty}} \\
& \times{ }_{2} \phi_{1}\left[\begin{array}{c}
\beta / z^{2}, \beta \\
q / z^{2} ; q, q^{\alpha}
\end{array}\right] \\
& \times{ }_{3} \phi_{2}\left[\begin{array}{c}
\beta z^{2}, \beta, z t \\
q z^{2}, \lambda z t
\end{array} ; q, q^{\alpha+1}\right] \\
+ & z \longleftrightarrow z^{-1} .
\end{aligned}
$$

It also follows from (3.5) that

$$
\begin{aligned}
\sum_{n=0}^{\infty} C_{n+k}^{\alpha}(x ; \beta \mid q) t^{n}= & \frac{\left(1-q^{\alpha}\right)}{\left(1-z^{-2}\right)} z_{2}^{k} \phi_{1}\left[\begin{array}{c}
\beta / z^{2}, \beta \\
q / z^{2}
\end{array} ; q, q^{\alpha}\right] \\
& \times{ }_{3} \phi_{2}\left[\begin{array}{c}
q z^{2} / \beta, q / \beta, z t \\
q z^{2}, q z t \quad ; q, \beta^{2} q^{\alpha+1}
\end{array}\right] \\
& +z \longleftrightarrow z^{-1}, \quad k=0,1, \ldots
\end{aligned}
$$

\section{Some bilinear generating functions for $C_{n}^{\alpha}(x ; \beta \mid q)$}

In this section, we shall compute a few bilinear generating functions. First, let us consider the simplest one:

$$
L_{t}^{\alpha}(x, y):=\sum_{n=0}^{\infty} \frac{\left(q^{\alpha+1} ; q\right)_{n}}{\left(\beta^{2} q^{\alpha} ; q\right)_{n}}\left(t q^{-\alpha}\right)^{n} C_{n}^{\alpha}(x ; \beta \mid q) C_{n}^{\alpha}(y ; \beta \mid q),
$$

which is symmetric in $x$ and $y$ where $x=\cos \theta, y=\cos \varphi, 0<\theta, \varphi<\pi, e^{i \theta}=z_{1}$, and $e^{i \varphi}=z_{2}$. By assuming $\left|t q^{-\alpha}\right|<1$ and using (4.3) in (5.1), we find that

$$
\begin{aligned}
L_{t}^{\alpha}(x, y)= & \frac{\left(1-q^{\alpha}\right)}{\left(1-z_{1}^{-2}\right)} \frac{\left(q^{\alpha+1} ; q\right)_{\infty}}{\left(\beta^{2} q^{\alpha} ; q\right)_{\infty}}{ }_{2} \phi_{1}\left[\begin{array}{c}
\beta / z_{1}^{2}, \beta \\
q / z_{1}^{2}
\end{array} ; q, q^{\alpha}\right] \\
& \times \sum_{k=0}^{\infty} \frac{\left(\beta z_{1}^{2}, \beta ; q\right)_{k}}{\left(q, q z_{1}^{2} ; q\right)_{k}} q^{(\alpha+1) k} \sum_{n=0}^{\infty}\left(z_{1} t q^{-\alpha+k}\right)^{n} C_{n}^{\alpha}(y ; \beta \mid q) \\
& +z_{1} \longleftrightarrow z_{1}^{-1} .
\end{aligned}
$$

However, by (1.17),

$$
\begin{aligned}
\sum_{n=0}^{\infty}\left(z_{1} t q^{-\alpha+k}\right)^{n} C_{n}^{\alpha}(y ; \beta \mid q)= & \frac{\left(1-q^{\alpha}\right)}{\left(1-2 y t z_{1} q^{k-\alpha}+z_{1}^{2} t^{2} q^{2 k-2 \alpha}\right)} \\
& \times{ }_{3} \phi_{2}\left[\begin{array}{l}
q, \beta t z_{1} q^{k-\alpha} e^{i \varphi}, \beta t z_{1} q^{k-\alpha} e^{-i \varphi} \\
t z_{1} q^{k-\alpha+1} e^{i \varphi}, t z_{1} q^{k-\alpha+1} e^{-i \varphi} ; q, q^{\alpha}
\end{array}\right]
\end{aligned}
$$


and so the sum over $k$ in (5.2) is

$$
\begin{aligned}
& \left(1-q^{\alpha}\right) \sum_{k=0}^{\infty} \sum_{j=0}^{\infty} \frac{\left(\beta z_{1}^{2}, \beta ; q\right)_{k}}{\left(q, q z_{1}^{2} ; q\right)_{k}} q^{(\alpha+1) k+\alpha j} \\
& \times \frac{\left(\beta t z_{1} q^{-\alpha} e^{i \varphi}, \beta t z_{1} q^{-\alpha} e^{-i \varphi} ; q\right)_{j+k}}{\left(t z_{1} q^{-\alpha} e^{i \varphi}, t z_{1} q^{-\alpha} e^{-i \varphi} ; q\right)_{j+k+1}} \frac{\left(t z_{1} q^{-\alpha} e^{i \varphi}, t z_{1} q^{-\alpha} e^{-i \varphi} ; q\right)_{k}}{\left(\beta t z_{1} q^{-\alpha} e^{i \varphi}, \beta t z_{1} q^{-\alpha} e^{-i \varphi} ; q\right)_{k}} \\
& =\frac{\left(1-q^{\alpha}\right)}{\left(1-2 y t z_{1} q^{-\alpha}+z_{1}^{2} t^{2} q^{-2 \alpha}\right)^{3}} \phi_{2}\left[\begin{array}{l}
q, \beta t z_{1} q^{-\alpha} e^{i \varphi}, \beta t z_{1} q^{-\alpha} e^{-i \varphi} \\
t z_{1} q^{-\alpha+1} e^{i \varphi}, t z_{1} q^{-\alpha+1} e^{-i \varphi} ; q, q^{\alpha}
\end{array}\right] \\
& \times{ }_{4} \phi_{3}\left[\begin{array}{l}
\beta, \beta z_{1}^{2}, t z_{1} q^{-\alpha} e^{i \varphi}, t z_{1} q^{-\alpha} e^{-i \varphi} \\
\left.q z_{1}^{2}, \beta t z_{1} q^{-\alpha} e^{i \varphi}, \beta t z_{1} q^{-\alpha} e^{-i \varphi} ; q, q\right] .
\end{array}\right.
\end{aligned}
$$

Hence,

$$
\begin{aligned}
L_{t}^{\alpha}(x, y)= & \frac{\left(1-q^{\alpha}\right)^{2}}{\left(1-z^{-2}\right)\left(1-2 y t z_{1} q^{-\alpha}+z_{1}^{2} t^{2} q^{-2 \alpha}\right)} \frac{\left(q^{\alpha+1} ; q\right)_{\infty}}{\left(\beta^{2} q^{\alpha} ; q\right)_{\infty}} \\
& \times{ }_{2} \phi_{1}\left[\begin{array}{c}
\beta / z_{1}^{2}, \beta \\
\left.q / z_{1}^{2} ; q, q^{\alpha}\right]
\end{array}\right. \\
& \times{ }_{3} \phi_{2}\left[\begin{array}{l}
q, \beta t z_{1} q^{-\alpha} e^{i \varphi}, \beta t z_{1} q^{-\alpha} e^{-i \varphi} \\
t z_{1} q^{-\alpha+1} e^{i \varphi}, t z_{1} q^{-\alpha+1} e^{-i \varphi} ; q, q^{\alpha}
\end{array}\right] \\
& \times{ }_{4} \phi_{3}\left[\begin{array}{l}
\beta, \beta z_{1}^{2}, t z_{1} q^{-\alpha} e^{i \varphi}, t z_{1} q^{-\alpha} e^{-i \varphi} \\
q z_{1}^{2}, \beta t z_{1} q^{-\alpha} e^{i \varphi}, \beta t z_{1} q^{-\alpha} e^{-i \varphi} ; q, q
\end{array}\right] \\
+ & z_{1} \longleftrightarrow z_{1}^{-1} .
\end{aligned}
$$

This leads to the expression (1.18) for the Poisson kernel for $C_{n}^{\alpha}(y ; \beta \mid q)$. Note that the moment representation of $C_{n}^{\alpha}(y ; \beta \mid q)$ also enables us to compute the non-symmetric kernel

$$
\begin{aligned}
M_{t}^{\alpha}\left(x, y ; \beta, \beta_{1}\right):= & \sum_{n=0}^{\infty} \frac{\left(q^{\alpha+1} ; q\right)_{n}}{\left(\beta^{2} q^{\alpha} ; q\right)_{n}}\left(t q^{-\alpha}\right)^{n} C_{n}^{\alpha}(x ; \beta \mid q) C_{n}^{\alpha}\left(y ; \beta_{1} \mid q\right) \\
= & \frac{\left(1-q^{\alpha}\right)^{2}}{\left(1-z^{-2}\right)\left(1-2 y t z_{1} q^{-\alpha}+z_{1}^{2} t^{2} q^{-2 \alpha}\right)} \frac{\left(q^{\alpha+1} ; q\right)_{\infty}}{\left(\beta^{2} q^{\alpha} ; q\right)_{\infty}} \\
& \times{ }_{2} \phi_{1}\left[\begin{array}{c}
\beta / z_{1}^{2}, \beta \\
q / z_{1}^{2}
\end{array} ; q, q^{\alpha}\right] \\
& \times{ }_{3} \phi_{2}\left[\begin{array}{c}
q, \beta_{1} t z_{1} q^{-\alpha} e^{i \varphi}, \beta_{1} t z_{1} q^{-\alpha} e^{-i \varphi} \\
\left.t z_{1} q^{-\alpha+1} e^{i \varphi}, t z_{1} q^{-\alpha+1} e^{-i \varphi} ; q, q^{\alpha}\right]
\end{array}\right. \\
& \times{ }_{4} \phi_{3}\left[\begin{array}{c}
\beta, \beta z_{1}^{2}, t z_{1} e^{i \varphi} q^{-\alpha}, t z_{1} e^{-i \varphi} q^{-\alpha} \\
\left.q z_{1}^{2}, \beta_{1} t z_{1} e^{i \varphi} q^{-\alpha}, \beta_{1} t z_{1} e^{-i \varphi} q^{-\alpha} ; q, q\right]
\end{array}\right. \\
+ & z_{1} \longleftrightarrow z_{1}^{-1} .
\end{aligned}
$$

Observe that the ${ }_{4} \phi_{3}$ series in (5.5) is balanced, but the one in (5.6) is not unless $\beta_{1}^{2}=\beta^{2}$. (5.3) may be seen as an extension of (5.5) of [11]. 
Finally, use of (3.5) and (1.17) gives

$$
\begin{aligned}
& \sum_{n=0}^{\infty} C_{n}^{\alpha}(x ; \beta \mid q) C_{n}^{\alpha}\left(y ; \beta_{1} \mid q\right) t^{n}=\frac{\left(1-q^{\alpha}\right)^{2}}{\left(1-z^{-2}\right)\left(1-2 y t z_{1}+z_{1}^{2} t^{2}\right)} \\
& \times{ }_{2} \phi_{1}\left[\begin{array}{c}
\beta / z_{1}^{2}, \beta \\
q / z_{1}^{2}
\end{array} ; q, q^{\alpha}\right] \\
& \times{ }_{3} \phi_{2}\left[\begin{array}{c}
q, \beta_{1} t z_{1} e^{i \varphi}, \beta_{1} t z_{1} e^{-i \varphi} \\
q t z_{1} e^{i \varphi}, q t z_{1} e^{-i \varphi} ; q, q^{\alpha}
\end{array}\right]
\end{aligned}
$$

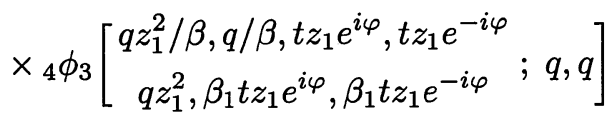

$$
\begin{aligned}
& +z_{1} \longleftrightarrow z_{1}^{-1} \text {. }
\end{aligned}
$$

\section{Some limiting formulas}

We will compute the $q \rightarrow 1$ limits of formulas (3.4) and (5.5). We believe that these formulas are new.

It is clear from the expressions on the right-hand side of (3.4) and (5.5) that one cannot take the term-by-term limits. One has to do some transformations of the $q$ series involved in these formulas. Using (III. 1) and (III. 3) of [7], in that order, we find that

$$
{ }_{2} \phi_{1}\left[\begin{array}{c}
q / \beta z^{2}, q / \beta \\
q / z^{2} ; q, \beta^{2} q^{\alpha-1}
\end{array}\right]=\frac{\left(\beta q^{\alpha}, \beta / z^{2} ; q\right)_{\infty}}{\left(\beta^{2} q^{\alpha-1}, q / z^{2} ; q\right)_{\infty}}{ }_{2} \phi_{1}\left[\begin{array}{c}
q^{\alpha}, q / \beta \\
\beta q^{\alpha}
\end{array} ; q, \beta / z^{2}\right]
$$

and

$$
{ }_{2} \phi_{1}\left[\begin{array}{c}
q z^{2} / \beta, q / \beta \\
q z^{2} ; q, \beta^{2} q^{\alpha+n}
\end{array}\right]=\frac{\left(\beta q^{\alpha+n+1}, \beta z^{2} ; q\right)_{\infty}}{\left(\beta^{2} q^{\alpha+n}, q z^{2} ; q\right)_{\infty}} \phi_{1}\left[\begin{array}{c}
q^{\alpha+n+1}, q / \beta \\
\beta q^{\alpha+n+1}
\end{array} ; q, \beta z^{2}\right]
$$

These two formulas along with the definition of the $q$-gamma function [7, (1.10.1)] give

$$
\begin{aligned}
C_{n}^{\alpha}\left(x ; q^{\lambda} \mid q\right)= & \frac{\Gamma_{q}(\alpha+1) \Gamma_{q}(2 \lambda+\alpha+n)}{\Gamma_{q}(\alpha+\lambda) \Gamma_{q}(\alpha+n+\lambda+1)} \frac{\left(z^{2} q^{\lambda}, q^{\lambda} / z^{2} ; q\right)_{\infty}}{\left(q z^{2}, q / z^{2} ; q\right)_{\infty}} \frac{z^{n}}{\left(1-z^{-2}\right)} \\
& \times{ }_{2} \phi_{1}\left[\begin{array}{c}
q^{\alpha}, q^{1-\lambda} \\
q^{\alpha+\lambda}
\end{array} ; q, q^{\lambda} / z^{2}\right]{ }_{2} \phi_{1}\left[\begin{array}{c}
q^{\alpha+n+1}, q^{1-\lambda} \\
q^{\alpha+n+1+\lambda}
\end{array} ; q, z^{2} q^{\lambda}\right] \\
& +z \longleftrightarrow z^{-1},
\end{aligned}
$$

where it is assumed that $\operatorname{Re}(\lambda)>0$ and $\alpha \geq 0$.

So the associated ultraspherical polynomials have the representation:

$$
\begin{aligned}
C_{n}^{\lambda, \alpha}(x)= & \frac{\Gamma(\alpha+1) \Gamma(2 \lambda+\alpha+n)}{\Gamma(\alpha+\lambda) \Gamma(\alpha+n+\lambda+1)} \frac{2^{2-2 \lambda} e^{i(n+1) \theta}}{2 i(\sin \theta)^{2 \lambda-1}} \\
& \quad \times{ }_{2} F_{1}\left[\begin{array}{c}
\alpha, 1-\lambda \\
\alpha+\lambda
\end{array} ; e^{-2 i \theta}\right]{ }_{2} F_{1}\left[\begin{array}{c}
\alpha+n+1,1-\lambda \\
\alpha+n+1+\lambda
\end{array} ; e^{2 i \theta}\right] \\
& +\theta \longleftrightarrow-\theta .
\end{aligned}
$$


For convergence on the unit circle of the two Gaussian series on the right-hand side we need the stronger condition $\operatorname{Re}(\lambda)>\frac{1}{2}$. One can rewrite (6.4) in the form

$$
\begin{aligned}
C_{n}^{\lambda, \alpha}(x) & =\frac{\Gamma(\alpha+1) \Gamma(2 \lambda+\alpha+n)}{\Gamma(\alpha+\lambda) \Gamma(\alpha+n+\lambda+1)} \frac{2^{2-2 \lambda}}{(\sin \theta)^{2 \lambda-1}} \\
& \times \sum_{j=0}^{\infty} \sum_{k=0}^{\infty} \frac{(\alpha)_{j}(1-\lambda)_{j}(\alpha+n+1)_{k}(1-\lambda)_{k}}{j ! k !(\alpha+\lambda)_{j}(\alpha+n+1+\lambda)_{k}} \sin (n+1-2 j+2 k) \theta,
\end{aligned}
$$

which is a generalization of $[21,(4.9 .22)]$ to which it reduces when $\alpha=0$. In order to find the $q \rightarrow 1$ limit of $L_{t}^{\alpha}(x, y)$, we do not use the final formula (5.5); rather we use the intermediate formula (5.2). First, by [7, (III.1)]

$$
{ }_{2} \phi_{1}\left[\begin{array}{c}
\beta / z_{1}^{2}, \beta \\
q / z_{1}^{2}
\end{array} ; q, q^{\alpha}\right]=\frac{\left(\beta q^{\alpha}, \beta / z_{1}^{2} ; q\right)_{\infty}}{\left(q^{\alpha}, q / z_{1}^{2} ; q\right)_{\infty}}{ }_{2} \phi_{1}\left[\begin{array}{c}
q^{\alpha}, q / \beta \\
\beta q^{\alpha}
\end{array} ; q, \beta / z_{1}^{2}\right] .
$$

Now, the series over $k$ in $(5.2)$ is

$$
\begin{aligned}
\sum_{n=0}^{\infty}\left(z_{1} t q^{-\alpha}\right)^{n} C_{n}^{\alpha}(y ; \beta \mid q)_{2} \phi_{1}\left[\begin{array}{c}
\beta z_{1}^{2}, \beta \\
q z_{1}^{2}
\end{array} ; q, q^{\alpha+n+1}\right] \\
=\frac{\left(\beta q^{\alpha+1}, \beta z_{1}^{2} ; q\right)_{\infty}}{\left(q^{\alpha+1}, q z_{1}^{2} ; q\right)_{\infty}} \sum_{n=0}^{\infty} \frac{\left(q^{\alpha+1} ; q\right)_{n}}{\left(\beta q^{\alpha+1} ; q\right)_{n}}\left(z_{1} t q^{-\alpha}\right)^{n} C_{n}^{\alpha}(y ; \beta \mid q) \\
\quad \times{ }_{2} \phi_{1}\left[\begin{array}{c}
q^{\alpha+n+1}, q / \beta \\
\beta q^{\alpha+n+1} ; q, \beta z_{1}^{2}
\end{array}\right] .
\end{aligned}
$$

We now substitute (6.5) and (6.6) into (5.2). and replace $\beta$ by $q^{\lambda}$ to get

$$
\begin{aligned}
& L_{t}^{\alpha}(x, y)=\frac{\Gamma_{q}(\alpha+1) \Gamma_{q}(2 \lambda+\alpha)}{\Gamma_{q}(\alpha+\lambda) \Gamma_{q}(\alpha+\lambda+1)} \frac{\left(z_{1}^{2} q^{\lambda}, q^{\lambda} / z_{1}^{2} ; q\right)_{\infty}}{\left(q z_{1}^{2}, q / z_{1}^{2} ; q\right)_{\infty}}\left(1-z_{1}^{-2}\right)^{-1} \\
& \times{ }_{2} \phi_{1}\left[\begin{array}{cc}
q^{\alpha}, q^{1-\lambda} \\
q^{\alpha+\lambda} & ; q, q^{\lambda} / z_{1}^{2}
\end{array}\right] \\
& \times \sum_{n=0}^{\infty} \frac{\left(q^{\alpha+1} ; q\right)_{n}}{\left(q^{\alpha+\lambda+1} ; q\right)_{n}}\left(z_{1} t q^{-\alpha}\right)^{n} C_{n}^{\alpha}\left(y ; q^{\lambda} \mid q\right)_{2} \phi_{1}\left[\begin{array}{c}
q^{\alpha+n+1}, q^{1-\lambda} \\
q^{\alpha+\lambda+n+1}
\end{array} ; q, z_{1}^{2} q^{\lambda}\right] \\
& +z_{1} \longleftrightarrow z_{1}^{-1}
\end{aligned}
$$

for $\operatorname{Re}(\lambda)>0, \operatorname{Re}(\alpha) \geq 0$. We now take the limit to obtain

$$
\begin{aligned}
\lim _{q \rightarrow 1^{-}} L_{t}^{\alpha}(x, y)= & \frac{\Gamma(\alpha+1) \Gamma(2 \lambda+\alpha)}{\Gamma(\alpha+\lambda) \Gamma(\alpha+\lambda+1)} \frac{2^{2-2 \lambda}}{(\sin \theta)^{2 \lambda-1}} \frac{e^{i \theta}}{2 i}{ }_{2} F_{1}\left[\begin{array}{c}
\alpha, 1-\lambda \\
\alpha+\lambda
\end{array} ; e^{-2 i \theta}\right] \\
& \times \sum_{n=0}^{\infty} \frac{(\alpha+1)_{n}}{(\alpha+\lambda+1)_{n}}\left(t e^{i \theta}\right)^{n} C_{n}^{\lambda, \alpha}(y)_{2} F_{1}\left[\begin{array}{c}
\alpha+n+1,1-\lambda \\
\alpha+n+1+\lambda
\end{array} ; e^{2 i \theta}\right] \\
& +\theta \longleftrightarrow-\theta .
\end{aligned}
$$

To ensure convergence of the two ${ }_{2} F_{1}$ series on the right-hand side, we assume, as before, that $\operatorname{Re}(\lambda)>\frac{1}{2}$. 
We now shall express the series over $n$ on the right-hand side of (6.8) as a double integral. First, by Euler's integral representation [7, (1.11.10)], we have

$$
\begin{aligned}
& \frac{\Gamma(\alpha+n+1) \Gamma(\lambda)}{\Gamma(\alpha+1+n+\lambda)}{ }_{2} F_{1}\left[\begin{array}{c}
\alpha+n+1,1-\lambda \\
\alpha+n+1+\lambda
\end{array} ; e^{2 i \theta}\right] \\
& \quad=\int_{0}^{1} u^{\alpha+n}(1-u)^{\lambda-1}\left(1-u e^{2 i \theta}\right)^{\lambda-1} d u, \quad 0<\theta<\pi, n=0,1, \ldots .
\end{aligned}
$$

Since

$$
\frac{\alpha}{\alpha+k}=\alpha \int_{0}^{1} v^{\alpha+k-1} d v, \quad \operatorname{Re}(\alpha)>0
$$

and, by $[6,(3.13)]$,

$$
C_{n}^{\lambda, \alpha}(y)=\sum_{k=0}^{n} \frac{\alpha}{\alpha+k} C_{n-k}^{\lambda}(y) C_{k}^{1-\lambda}(y)
$$

we find that

$$
\begin{aligned}
& \frac{\Gamma(\alpha+1)}{\Gamma(\alpha+1+\lambda)} \sum_{n=0}^{\infty} \frac{(\alpha+1)_{n}}{(\alpha+\lambda+1)_{n}}\left(t e^{i \theta}\right)^{n} C_{n}^{\lambda, \alpha}(y)_{2} F_{1}\left[\begin{array}{c}
\alpha+n+1,1-\lambda \\
\alpha+n+1+\lambda
\end{array} ; e^{2 i \theta}\right] \\
& =\frac{\alpha}{\Gamma(\lambda)} \int_{0}^{1} \int_{0}^{1} u^{\alpha}(1-u)^{\lambda-1}\left(1-u e^{2 i \theta}\right)^{\lambda-1} v^{\alpha-1} \\
& \quad \times\left\{\sum_{n=0}^{\infty}\left(u t e^{i \theta}\right)^{n} \sum_{k=0}^{n} C_{n-k}^{\lambda}(y) C_{k}^{1-\lambda}(y) v^{k}\right\} d u d v \\
& =\frac{\alpha}{\Gamma(\lambda)} \int_{0}^{1} \int_{0}^{1} u^{\alpha}(1-u)^{\lambda-1}\left(1-u e^{2 i \theta}\right)^{\lambda-1} v^{\alpha-1}\left(1-2 u t y e^{i \theta}+u^{2} t^{2} e^{2 i \theta}\right)^{-\lambda} \\
& \times\left(1-2 u v t y e^{i \theta}+u^{2} v^{2} t^{2} e^{2 i \theta}\right)^{\lambda-1} d u d v,
\end{aligned}
$$

where the last line has been obtained by using the well-known generating function of the ultraspherical polynomials. Thus, we get

$$
\begin{aligned}
\lim _{q \rightarrow 1^{-}} L_{t}^{\alpha}(x, y)= & \frac{\alpha \Gamma(2 \lambda+\alpha)}{\Gamma(\alpha+\lambda) \Gamma(\lambda)} \frac{2^{2-2 \lambda}}{(\sin \theta)^{2 \lambda-1}} \frac{e^{i \theta}}{2 i}{ }^{2} F_{1}\left[\begin{array}{c}
\alpha, 1-\lambda \\
\alpha+\lambda
\end{array} ; e^{-2 i \theta}\right] \\
& \times \int_{0}^{1} \int_{0}^{1} u^{\alpha}(1-u)^{\lambda-1}\left(1-u e^{2 i \theta}\right)^{\lambda-1} v^{\alpha-1}\left(1-2 u t y e^{i \theta}+u^{2} t^{2} e^{2 i \theta}\right)^{-\lambda} \\
& \times\left(1-2 u v t y e^{i \theta}+u^{2} v^{2} t^{2} e^{2 i \theta}\right)^{\lambda-1} d u d v \\
& +\theta \longleftrightarrow-\theta,
\end{aligned}
$$

with $|t|<1$. Using (6.13), one can easily find the limit of $K_{t}^{\alpha}(x, y)$.

Acknowledgement. The work of the first author was supported in part by an NSERC grant A6197. This is part of the second author's dissertation in partial fulfillment of the requirements of his doctoral program at Carleton University. We would like to thank the referee for drawing our attention to reference [13] as well as for making suggestions that led to some substantial improvements on the original manuscript. 


\section{References}

1. R. A. Askey, Orthogonal Polynomials and Special Functions, Regional Conference Series in Applied Mathematics 21, SIAM, Philadelphia, 1975.

2. R. A. Askey, N. M. Atakishiyev, and S. K. Suslov, An analogue of the Fourier transformation for the q-harmonic oscillator, preprint IAE-5611/1, Kurchatov Institute, Moscow; Symmetries in Sciences 6 (Ed. B. Gruber), Plenum Press, New York, 1993, pp. 57-63.

3. R. A. Askey and M. E. H. Ismail, A generalization of ultraspherical polynomials, In: Studies in Pure Mathematics, (Ed. P. Erdös), Birkhauser, Basel, 1983, pp. 55-78.

4. R. A. Askey, M. Rahman, and S. K. Suslov, On a general q-Fourier transformation with nonsymmetric Kernel, J. Comp. Appl. Math. 68 (1996), 25-55.

5. R. A. Askey and J. A. Wilson, Some basic hypergeometric orthogonal polynomials that generalize Jacobi polynomials, Memoirs Amer. Math. Soc. 319, 1985.

6. J. Bustoz and M. E. H. Ismail, The associated ultraspherical polynomials and their q-analogues, Canadian J. Math. 34 (1982), 718-736.

7. G. Gasper and M. Rahman, Basic Hypergeometric Series, Cambridge University Press, Cambridge, 1990.

8. - Positivity of the Poisson kernel for the continuous q-ultraspherical polynomials, SIAM J. Math. Anal. 14 (1983), 409-420.

9. __ Positivity of the Poisson kernel for the continuous $q$-Jacobi polynomials and some quadratic transformation formulas for basic hypergeometric series, SIAM J. Math. Anal. 17 (1986), 970-999.

10. M. E. H. Ismail and M. Rahman, The associated Askey-Wilson polynomials, Trans. Amer. Math. Soc. 328 (1991), 201-237.

11. M. E. H. Ismail and D. Stanton, Classical orthogonal polynomials as moments, Canadian J. Math., to appear.

12. More orthogonal polynomials as moments, to appear.

13. S. Karlin, Sign regularity of classical orthogonal polynomials, in: Orthogonal Expansions and Their Continuous Analogue, (Ed. D. Haimo), Southern Illinois University Press, Carbondale, 1967, pp. 55-74.

14. M. Rahman, Series and integral representation of $C_{n}^{2}(x ; \beta \mid q)+D_{n}^{2}(x ; \beta \mid q)$, J. Math. Anal. Appl. 164 (1992), 263-284.

15. M. Rahman and S. K. Suslov, Singular analogue of the Fourier transformation for the AskeyWilson polynomials, Centre de Recherches Mathematiques, CRM Proceedings and Lecture Notes 9, 1996, pp. 289-302.

16. M. Rahman and A. Verma, Positivity of the Poisson kernel for the Askey-Wilson polynomials, Indian J. Math. 33 (1991), 287-306.

17. L. J. Rogers, On the three-fold symmetry in the elements of Heine's series, Proc. London. Math. Soc. 24 (1893), 171-179.

18. Second memoir on the expansion of certain infinite products, Proc. London. Math. Soc. 25 (1894), 318-343.

19. - Third memoir on the expansion of certain infinite products, Proc. London. Math. Soc. 26 (1895), 15-32.

20. - On the expansion of some infinite products, Proc. London. Math. Soc. 24 (1893), 337352.

21. G. Szegö, Orthogonal Polynomials, 4th ed., AMS Coll Pub. 23, American Mathematical Society, Providence, R.I., 1975.

22. G. N. Watson, Notes on generating functions of polynomials, Proc. London. Math. Soc. 8 (1933), 194-199.

23. N. Wiener, The Fourier Integral and Certain of Its Applications, Cambridge University Press, Cambridge, 1933; Dover edition published in 1948.

Department of Mathematics and Statistics, Carleton University, Ottawa, Ontario K1S 5B6, CANADA

E-mail: mrahman@math.carleton.ca, qtariq@mathstat.carleton.ca 